\title{
Analysis of corrosion risk due to chloride diffusion for concrete structures in marine environment
}

\author{
Tristan Senga Kiesse ${ }^{c}$, Stéphanie Bonnet ${ }^{b, *}$, Ouali Amiri ${ }^{b}$, Anne Ventura ${ }^{\text {a,d }}$ \\ ${ }^{a}$ UBL, Université de Nantes, GeM UMR CNRS 6183, Institut de recherche en Génie Civil et Mécanique, Chaire Génie Civil et Ecoconstruction, IUT \\ Saint-Nazaire, 52 rue Michel Ange, BP 420, Saint-Nazaire cedex, France \\ ${ }^{\mathrm{b}}$ UBL, Université de Nantes, GeM CNRS UMR 6183, Institut de recherche en Génie Civil et Mécanique, IUT Saint-Nazaire, 52 rue Michel Ange, BP \\ 420, Saint-Nazaire cedex, France \\ ${ }^{\mathrm{c}}$ UMR SAS, INRAE, Agrocampus Ouest, 35000, Rennes, France \\ d Institut Français Transports Aménagement Réseaux (IFSTTAR - MAST - GPEM), France
}

The chloride-induced steel corrosion is one of the main causes of deterioration for reinforced concrete structures exposed to marine environments. The chloride ingress into reinforced con-crete structures is even more complex since it depends on random parameters linked to transport and chemical properties of materials, which results in variability of corrosion initiation. This variation raises the need of statistical approaches to evaluate the risk of corrosion initiation due to chloride ingress. To address this issue, we use sensitivity analysis to identify the influence of input parameters on critical length of time before corrosion initiation predicted by our chloride diffusion model. Exceedance probabilities of corrosion initiation time given that input parameters exceed certain thresholds were also calculated. Results showed that the corrosion initiation time was most sensitive to: chloride effective diffusion coefficient $D_{e}$ in concrete, that is a parameter controllable by relevant stakeholders; surface chloride concentration $C_{s}$, a non-controllable parameter depending on surrounding conditions. Reducing the chloride diffusion coefficient enables us to postpone the maintenance of structures. However, the interaction between controllable parameters and noncontrollable surrounding conditions was revealed influential on the reliability of results. For instance, the probability that corrosion initiation time exceeds 15 years given an effective diffusion coefficient $\left(D_{e}\right)$ equal to $0.1 \times 10^{-12} \mathrm{~m}^{2} \cdot \mathrm{s}-1$ can vary from 19 to $41 \%$ according to stochastic variations of chloride concentrations $\left(C_{s}\right)$ values. Postponing the corrosion initiation time was combined with a decreasing probability of its occurrence.

\section{Introduction}

Corrosion of steel reinforcement due to chloride ingress is one of the major causes of degradation of Reinforced Concrete (RC) structures [1]. According to Tuutti diagram [2], the corrosion can be divided into two stages: corrosion initiation and corrosion propagation. The corrosion initiation corresponds to the process of chloride ingress into concrete until the chloride concentration has reached the steel rebar and exceeds a threshold value. Then steel is de-passivated and the corrosion propagates into the reinforcement. We define the service life as the period of penetration of chloride into the concrete cover until the chloride content exceeds a threshold

\footnotetext{
* Corresponding author.

E-mail address: stephanie.bonnet@univ-nantes.fr (S. Bonnet).
} 
value at the position of the reinforced steel bar. Indeed, at the end of that period, maintenance operations are required: most current maintenance operations consist in removing the chloride contaminated concrete and replacing it by a new one [3], thus inducing additional costs mainly caused by concrete production. There is thus a balance to ensure both long service life and minimum costs. In a broader perspective, we can also consider that there should also be a balance for environmental impacts because cement concrete is an important contributor to climate change [4]. Consequently, it is important to improve service life predictions, but also to determine influent parameters and to evaluate levels of potential risk in order to provide recommendations for longer service life to engineering designers when designing concrete structure exposed to chloride.

Current studies are increasingly interested in reliability assessment of results on corrosion initiation from chloride ingress modelling. For instance, a probabilistic approach to assess chloride ingress into concrete was performed by analyzing a model of chloride penetration into a stochastic framework [5]. More precisely, this last study applied Monte Carlo simulations and Latin hypercube sampling to consider propagation of uncertainties related to material properties and surrounding conditions. Likewise, a probabilistic analysis of corrosion initiation time was performed by focusing on the initiation phase of chloride induced reinforcement corrosion, when assessing five concrete durability options [6]. Recently, probabilistic de-passivation time for RC structures exposed to chloride ingress was also estimated according to three simple diffusion models, based on experimental data from a concrete structure exposed to the atmospheric marine environment [7]. Meanwhile, probabilistic risk analyses were also conducted for other causes of corrosion of concrete structures, such as corrosion risk due to carbonation [8]. However, in these and other studies, the individual influence of model's input parameters and the influence of their interactions on chloride ingress phenomenon were not investigated. This is required to be deeply investigated, in addition to probabilistic analysis of corrosion initiation time.

Our research aims at combining service life prediction models in order to give recommendations to the design engineers for extending service life. This kind of approach was already developed and applied to a RC structure located in Madrid and submitted to carbonation [9]. In complement to this previous work, the present study focuses on probabilistic assessment of corrosion risk due to chloride ingress into RC structures exposed to marine environment. First, a model for chloride ingress into concrete is chosen based on appropriate simplification in order to use it as decision tool for design engineers. Several studies focused on modeling chloride ingress by exclusively considering the diffusion process or by considering diffusion and convection [10]. Our approach requires a simplified durability model based on parameters (i.e. concrete mix design, construction parameters) that are available and controllable by the engineering designer, and including as well as relevant (i.e. influent) non controllable parameters (meteorology, salinity, ...). Second, a probabilistic analysis to corrosion risk due to chloride ingress is performed. That includes a sensitivity analysis (SA) to identify individual influences of model's parameters and of their interaction on service life of marine RC structures.

Various SA studies have already been conducted on chloride ingress inside cement concrete. For example, Boddy et al. [11] applied a one at a time (OAT) technique for sensitivity investigation of a chloride transport model to the variations of the parameters controlling the rate of diffusion, temperature of exposure, critical chloride level, diffusion coefficient, permeability coefficient, surface chloride concentration and position of steel rebar. Likewise, Kirkpatrick et al. [12] studied a probabilistic model to predict chloride corrosion initiation depending on the position of steel rebar, surface chloride concentration and apparent diffusion coefficient. A deeper deterministic SA than the two previous studies was conducted by undertaking a differential analysis technique on corrosion model depending on four governing parameters: apparent chloride diffusion coefficient, position of steel rebar, surface chloride concentration, chloride threshold [13]. A global SA using Sobol method was used to study the sensitivity of the probability of failure to the assumed coefficients of variation of properties of the pre-stressed concrete [14]. Likewise, the Sobol method was applied to investigate independent and cooperative effects of the material parameters on masonry compressive strength [15]. In addition to SA, exceedance probabilities of model output are calculated given that input parameters exceed certain thresholds, for reliability assessment of service life improvement.

Our study proposes to apply successively Morris [16] and Sobol [17] approaches as complementary methods for SA of diffusion-based chlorides model. The originality of our work is to go beyond the recent studies in literature on uncertainties in the models describing the degradation of reinforced concrete structures, by considering the individual influence of available input parameters as well as the influence of their interaction on model output. A nonlinear chloride transport model easy to implement is used to enable us to investigate the potential interaction between input parameters, for which few experimental results are available in the literature. SA methods are applied to better understand the phenomena involved in chloride model and identifying input parameters (i. e. action levers) that sector's actors can control to improve service life behavior of concrete structures. These controllable (or technological) parameters are the action levers that will result in the proposition of possible pathways for structure owners to extend the length of time before corrosion initiation. Furthermore, the probabilistic analyses of the critical length of time to corrosion initiation given the most influent parameters are calculated to provide information on the reliability of results.

\section{Material and methods}

\subsection{Chloride transport model}

Various approaches were developed to model the chloride ingress through cement based materials. The main difference between models concern the fact that they considered concrete exposed in saturated and/or unsaturated conditions.

For saturated conditions, the diffusion process obeying the Fick's second law is generally sufficient to model the chloride ingress [18]. Likewise, the time-dependence of the apparent diffusion coefficient is considered as well as the chloride concentration of the exposed surface $[19,20]$ by using empirical laws to establish time dependency functions.

For models accounting for unsaturated conditions, some studies have modeled chloride transport by taking into account both 
diffusion and convection [21-27]. These models used semi-empirical laws found by fitting experimental data to get the moisture diffusivity and the chloride diffusion coefficient. These models can be mono-species (only chloride is considered) or multi-species (ions contained in the pore solution are also considered). They are considered as sophisticated models because they take into account some physical or chemical phenomena occurring into concrete such as chemical binding, electrical double layer, activity of pore solution.

However, models suitable for unsaturated conditions require many input parameters that are not currently measured for concrete design because they are expensive or time-demanding to collect and they are not required by the standards [28,29]. Moreover, reinforced concrete material in marine environment can generally be considered as saturated. Indeed, when casted on site, concrete is initially saturated and persistently exposed to high Relative Humidity (RH) that, for instance, stands superior to $80 \%$ on the French Atlantic coast [30]. In the case of maritime structures it is thus obvious to consider the chloride displacement into concrete by a diffusion equation as it was done by some studies to analyze chloride profiles obtained from reinforced concrete structures in unsaturated conditions [30-34]. From an engineer point of view, the Fick's second law is simple and the orders of magnitude of diffusion coefficient (or chloride profile), which we can obtain with this law, are correct and acceptable [35]. In addition, numerical implementation of this law is not time consuming in order to conduct statistical investigation.

Thus, according to our objective, our choice goes to a simple model suitable for saturated conditions. This model is resumed in Fig. 1. Equations are justified below.

Fick's second law of diffusion is written as:

$$
\frac{\partial C(x, t)}{\partial t}=D \frac{\partial^{2} C}{\partial x^{2}}
$$

where $C(x, t)\left(\mathrm{kg} \cdot \mathrm{m}^{-3}\right.$ of concrete) is the chloride concentration at position $\mathrm{x}(\mathrm{m})$ at time $\mathrm{t}(\mathrm{s})$.

The previous equation can be solved by considering finite or semi-infinite boundary conditions. The last case is often considered for computing diffusion coefficient or chloride concentration, because the unsteady state is not time consuming. By assuming a timeinvariant chloride concentration $C_{s}$ at the concrete surface and a time-invariant diffusion coefficient $D$, Crank's solution of second Fick's law is given by Crank [36]:

$$
C(x, t)=C_{s} \operatorname{erfc}\left(\frac{x}{2 \sqrt{D_{a} t}}\right),
$$

under the initial condition $C=0$ for $\mathrm{x}>0$ and $t=0$. In equation (2), $\mathrm{C}_{\mathrm{s}}\left(\mathrm{kg} \cdot \mathrm{m}^{-3}\right.$ of solution) is the chloride concentration at the concrete surface, $D_{a}\left(m^{2} \cdot s^{-1}\right)$ is the apparent diffusion coefficient and erfc() is the complementary error function.

Input parameters $C_{s}$ and $D_{a}$ are not time-dependent in this paper, as it was done by Ref. [31-33]. Moreover it was shown that the chloride diffusion coefficient decreases particularly in the first few months before being stabilized even with admixtures as slag [37].

The apparent diffusion coefficient $D_{a}$ previously mentioned is given as [1]:

$$
D_{a}=\frac{D_{e}}{1+\frac{1}{p} \frac{\partial C_{b}^{\prime}}{\partial C_{f}}}
$$

where $D_{e}\left(\mathrm{~m}^{2} \cdot \mathrm{s}^{-1}\right)$ is the effective diffusion coefficient, $\mathrm{p}$ is the porosity and, $C_{b}^{\prime}\left(\mathrm{kg} \cdot \mathrm{m}^{-3}\right.$ of concrete) and $C_{f}\left(\mathrm{~kg} . \mathrm{m}^{-3}\right.$ of pore solution) are the concentrations of bound and free chlorides, respectively [38].

In order to easily use the statistical study, we have to note that as first step, we considered the diffusion coefficient as intrinsic

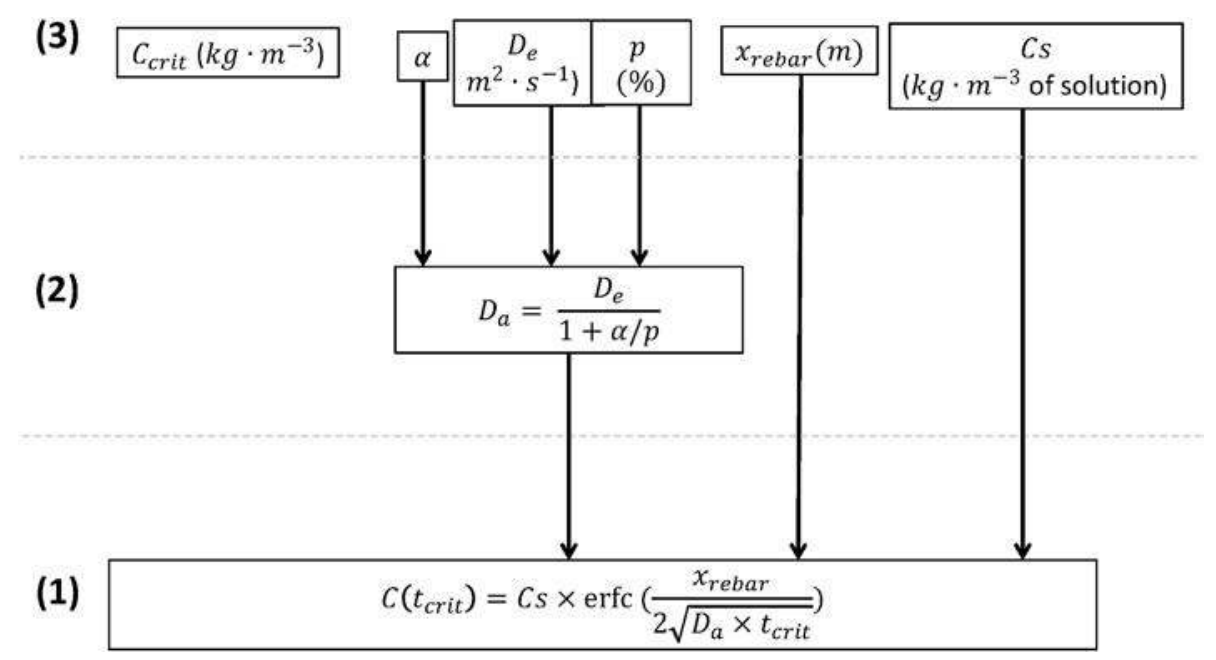

Fig. 1. Representation of chloride diffusion model: (1) model output, (2) intermediary model, (3) input parameters. 
property of the material so "constant". Taking into account the time dependency of the diffusion coefficient by introducing the ageing factor $[39,40]$ is a possible and interesting step not done in this paper.

Chloride binding isotherm is the relationship between free and bound chloride ions at a given temperature such that the partial derivative $\partial C_{b} / \partial C_{f}^{\prime}$ can be determined. The chloride binding capability of concrete is a very complex phenomena describing by thermodynamic equilibrium, kinetic control and surface complexation [41] and the effects of different types of chloride binding (physically and chemically bound chloride) can be taken into account to describe the chloride ingress [42]. Usually to evaluate the relationship $\partial C_{b}^{\prime} / \partial C_{f}$, three types of binding isotherms (linear, Langmuir, Freundlich) have been proposed by fitting isotherm models with measurements [43]. In order to experimentally determine this isotherm, researchers usually use powder or thin disc extracted from concrete cores. In that way the best fitting with experimental data was obtained with the Freundlich isotherm in many cases [37, 44]. However, this experimental procedure did not match the observations on reinforced concrete in a natural maritime environment. So, to establish the relationship between free and bound chloride, it is necessary to expose concrete specimens under marine environment for a long time [45,46]. For the different concrete compositions, which were tested in these studies, the obtained chloride binding capacity fitted very well with the linear relationship. So, the linear isotherm is used as:

$$
C_{b}^{\prime}=\alpha C_{f} \text {. }
$$

That results in the apparent diffusion coefficient given as follows:

$$
D_{a}=\frac{D_{e}}{1+\frac{\alpha}{p}} .
$$

In summary, the chloride diffusion model is presented in Fig. 1 with the main input parameters involved in chloride ingress into RC structures. We are interested in determining the critical time $t=t_{\text {crit }}$ that corresponds to the period of time during which chlorides penetrate the concrete but no damage is observed [2]:

$$
C\left(x_{\text {rebar }}, t_{\text {crit }}\right)=C_{\text {crit }} \text { for } x_{\text {rebar }}=\text { steel rebar position, }
$$

where the critical chloride concentration $C_{c r i t}\left(\mathrm{~kg} \cdot \mathrm{m}^{-3}\right.$ of concrete) is the chloride concentration threshold above which passivation of steel is destroyed [47].

The critical chloride concentration involved in equation (6) can take three forms [10]: the concentration of total chlorides [48], the concentration of free chlorides [49], and the $[\mathrm{Cl}] /[\mathrm{OH}]$ ratio [50]. These levels are highly variable because they depend on particular surrounding conditions, such as concrete mix design, experimental procedures [51], as well as rebar surface conditions [52,53]. The concentration of total chlorides was shown to be more relevant than the concentration of free chlorides alone or than the $[\mathrm{Cl}] /[\mathrm{OH}]$ ratio [54,55], thus we choose the total chloride content as our critical chloride concentration for the threshold value.

The critical length of time $t_{\text {crit }}$ to corrosion initiation is defined as the time at which the chloride concentration at the position of steel rebar $x_{\text {rebar }}$ exceeds the critical value. The time $t_{\text {crit }}$ can be analytically expressed as:

$$
t_{\text {crit }}=\frac{x_{\text {rebar }}{ }^{2}}{4 D_{a} \operatorname{erfc}^{-1}\left(\frac{C_{c r i t}}{C_{s}}\right)} .
$$

\subsection{Risk analysis study}

According to our objective, to obtain best service life values, a risk analysis study induced by chloride ingress phenomenon is conducted in three steps: 1 ) to perform SA to evaluate the influence of our model's various input parameters on its output parameters $[17], 2)$ to calculate conditional probabilities of critical length time to corrosion initiation, and 3) to analyze favorable and unfavorable scenarios to corrosion initiation.

\subsubsection{Sensitivity analysis}

At the first step, Morris and Sobol SA methods are applied to the model. The method of Morris [16] provides sensitivity information (influential, sense of variation) about input parameters on the interval range of interest. As a complement to the former, the method of Sobol [17] evaluates the contribution of the variation of each input parameter to the total variation of model output via quantitative indices. Sobol indices also quantify the contribution of the interaction between input parameters. Morris and Sobol methods are successively conducted based on the same methodology originated from Andrianandraina et al. [56]. More precisely, in this methodology, the model is first defined with used intermediary models, constants and input parameters. All input parameters are then characterized with their pdf and are grouped in two categories according to the actor's action possibility: technological that are controllable by the engineering designer and corresponding to potential action levers, and surrounding parameters that are not controllable by the engineering designer. Morris and Sobol methods are successively applied using variation range and pdf of parameters. More details on these two SA methods are provided in Supplementary Material (Section B).

\subsubsection{Conditional probability of critical length of time to corrosion initiation}

At the second step, conditional distributions of critical length of time $t_{\text {crit }}$ to corrosion initiation are calculated given the most influential input parameters. More precisely, that consists in evaluating exceedance probabilities of $t_{\text {crit }}$ given that input parameters $X_{1}$ 
and $X_{2}$ exceed certain thresholds $x_{1}$ and $x_{2}$, respectively, by setting other input parameters at their default or mean value, such as:

$$
\operatorname{Prob}\left(t_{c r i t} \geq t \mid X_{1} \geq x_{1}, X_{2} \geq x_{2}\right)=\frac{\operatorname{Prob}\left(t_{c r i t} \geq t, X_{1} \geq x_{1}, X_{2} \geq x_{2}\right)}{\operatorname{Prob}\left(X_{1} \geq x_{1}, X_{2} \geq x_{2}\right)}
$$

We will practically considered input parameters $X_{1}$ and $X_{2}$ having the most important contribution on variations of critical length of time $t_{\text {crit }}$.

\subsubsection{Comparisons of simulated scenarios}

At the third step, unfavorable, favorable and medium scenarios are simulated and compared. The first scenario called "unfavorable" aims at simulating the degradation of service life that the engineering designer can obtain by choosing values of parameters that decrease service life. Thus all influential technological parameters are set at their most unfavorable for the shortest service life and all surrounding parameters at their default or mean value [56]. The second scenario called "favorable" aims at simulating the reliability of the possible improvement of service life that the engineering designer can obtain by choosing values of parameters that increase service life. Thus all influential technological parameters are set at their most favorable for the longest service life and all surrounding parameters at their default or mean value [56]. The last scenario, called "medium" aims at obtaining an average scenario, for which values of parameters are not known and thus all set to their mean values. Probability distributions of simulated critical length of time $t_{c r i t}$ are then plotted according to the three previous scenarios. The comparison among probability distributions of $t_{c r i t}$ and their descriptive statistics (mean and standard deviation) will provide information about uncertainty and potential variation (extension or reduction) of the corrosion initiation time in each simulated scenario.

All calculations (SA indices, conditional probabilities, various scenarios) were realized over 5000 repetitions by Monte Carlo simulations. This number of repetitions was sufficient to reach stable and relevant results, with a reasonable computation time. Input parameters are assumed to be independent, as required in the methodology of the two SA methods applied.

\subsection{Characterization of input parameters}

According to our objective, we investigate the individual influence of input parameters $D_{e}, x_{\text {rebar }}, C_{s}, C_{\text {crit }}, p$ and $\alpha$, as well as the influence of their interaction on the corrosion initiation time $t_{\text {crit }}$.

Technological parameters are the position of steel rebar in concrete since it is governed by the European normalization (EN 1992-1-1 [29]), the effective diffusion coefficient $D_{e}$, the porosity $p$ because the engineering designer can control them through other factors such as the Water/Binder ratio. Surrounding parameters are the chloride concentration at the concrete surface, the linear isotherm $\alpha$, as well as the critical chloride content which it is sensitive to chemical characteristics of concrete components (binder and type of aggregates).

Probability density functions (pdf) of input parameters considered in this study are shown in Table 1 and we explain below how they were obtained.

Descriptive statistics of parameters related to properties of the materials (chloride diffusion, porosity) were calculated from a literature review of experimental studies performed on 13 references with 52 different concrete mixtures and different types of binders [37,57-67]. The references were selected because they determine the effective diffusion coefficient $D_{e}$ in concrete from accelerated steady state migration tests with a method closed to the French standard XP-P-18461. The distributions of observed values of effective diffusion coefficient $D_{e}$ and porosity $p$ obtained from our literature review were assumed lognormal and normal, respectively (Fig. S 1 in Supplementary Material). The influence of water/Binder ratios and incorporation of mineral admixtures, on porosity and diffusion coefficient, are described by Fig. S2 in Supplementary Material.

Concerning the surface chloride concentration $C_{s}$, the values corresponding to moderate and severe corrosive exposures were

Table 1

Input parameters for chloride diffusion model and their descriptive statistics. Column headings: parameter, unit, variable classification, mean, coefficient of variation (COV), probability density function (pdf), variation range, sources.

\begin{tabular}{|c|c|c|c|c|c|c|c|}
\hline Parameter & Unit & Class & Mean & $\begin{array}{l}\text { COV (standard } \\
\text { deviation) }\end{array}$ & Pdf & $\begin{array}{l}\text { Variation } \\
\text { range }\end{array}$ & Sources \\
\hline Porosity $(p)$ & $\%$ & Tech & 12.85 & $0.16(2)$ & Normal & $8.1-17.3$ & Fig. S 1 \\
\hline $\begin{array}{l}\text { Effective diffusion } \\
\text { coefficient }\left(D_{e}\right)\end{array}$ & $10^{-12} \mathrm{~m}^{2} \cdot \mathrm{s}^{-1}$ & Tech & 0.66 & $1.24(0.82)$ & Log-normal & $0.1-6.0$ & \\
\hline $\begin{array}{l}\text { Position of steel rebar } \\
\qquad\left(x_{\text {rebar }}\right)\end{array}$ & $\mathrm{Mm}$ & Tech & 50 & $0.25(12.5)$ & $\begin{array}{l}\text { Normal (truncated at lower bound } \\
=10 \mathrm{~mm} \text { ) }\end{array}$ & $10-75$ & [69] \\
\hline Linear isotherm $(\alpha)$ & n.u. & Surr & - & - & Uniform & $0.01-0,1$ & {$[45,46]$} \\
\hline $\begin{array}{l}\text { Surface Chloride } \\
\quad \text { Concentration }\left(\mathrm{C}_{\mathrm{s}}\right)\end{array}$ & $\begin{array}{l}\mathrm{kg} \cdot \mathrm{m}^{-3} \text { of pore } \\
\text { solution }\end{array}$ & Surr & 9.75 & $0.51(4.97)$ & $\begin{array}{l}\text { Normal (truncated at lower bound } \\
=1.67 \mathrm{~kg} \cdot \mathrm{m}^{-3} \text { ) }\end{array}$ & $1.67-17$ & {$[68]^{\mathrm{a}}$} \\
\hline $\begin{array}{l}\text { Critical chloride } \\
\quad \text { concentration }\left(C_{\text {crit }}\right)\end{array}$ & $\mathrm{kg} \cdot \mathrm{m}^{-3}$ of concrete & Surr & 0.9 & 0.19 & Uniform & $0.6-1.2$ & {$[68]^{b}$} \\
\hline
\end{tabular}

n.u = no unit; Tech: technological (controllable); Surr: surrounding conditions (not controllable).

a Table 2a in Ref. [68] (page 1607) presents descriptive statistics of surface concentration for structures exposed to marine breeze.

b Table 3 in Ref. [68] (page 1608) presents descriptive statistics of critical chloride concentration. 
considered for testing corrosion initiation and a normal distribution is assumed in this study [13,68]. More precisely, any conclusions were drawn about statistical distributions of $C_{s}$ for structures exposed to marine breeze [68]. In our work, we considered a normal distribution of $C_{s}$ truncated at the minimum value of its variation range.

The linear isotherm parameter $\alpha$ is experimental and it does not have a known pdf in literature. Some $\alpha$ values that were calculated in one study varied from 0.01452 to 0.06195 [45]. In that study, the concrete specimens were exposed to marine environment for 10-30 years before samples were collected to determine total and free chlorides content. The Water/Binder ratios varied from 0.45 to 0.55 and different binders were studied: ordinary Portland cement, slag cement and fly ash cement. Concrete specimens with Water/Binder ratios varying from 0.45 to 0.65 were also used with different binders (cement types CEMI, CEMV, as well as binders with fly ash replacing Portland cement type CEMI) [46]. In that study, the concrete specimens were exposed to seawater for seven years before determining total and free chloride content. The $\alpha$ values calculated from these data varied from 0.01584 to 0.0423 . From these two references we assume that the $\alpha$ parameter can vary inside the $[0.01 ; 0.1]$ interval. As we only have a minimum and maximum value, but no information on its pdf, we assume a uniform probability distribution.

The critical chloride concentration inducing the end of the de-passivation state [2], chosen as the concentration of total chlorides $[54,55]$ as previously explained, depends on many parameters such as the type of steel or electrochemical environment in concrete [68]. Its pdf being unknown, we also assume a uniform probability distribution.

At last, the position of steel rebar is assumed to follow a normal distribution truncated at a lower bound equal to $10 \mathrm{~mm}$ [69].

\section{Results and discussion}

\subsection{Sensitivity analysis}

Results of SA concerning the influence of input parameters $D_{e}, x_{\text {rebar }}, C_{s}, C_{c r i t}, p$ and $\alpha$ of the studied model are given in Table 2 .

\subsubsection{Individual influences}

According to the algebraic sign of Morris index $\mu$, the time $t_{\text {crit }}$ may be reduced $(\mu<0)$ by an increase of $D_{e}, p$ and $C_{s}$. Conversely, the time $t_{c r i t}$ may be extended $(\mu>0)$ by an increase of $C_{c r i t}, x_{\text {rebar }}$ and $\alpha$. These trends for $D_{e}, p$ and $\alpha$ are expected from the analytical expression of apparent diffusion coefficient $D_{a}$ in equation (5). Increasing $\alpha$ decreases the apparent diffusion $D_{a}$, while increasing $p$ increases $D_{a}$. Then, looking at the individual effect of parameters $\left(S_{i}\right)$ as well as the total effect $\left(S T_{i}\right)$, the time $t_{\text {crit }}$ is found most sensitive to variations of $D_{e}\left(S_{i} .=29.90 \%\right)$. That emphasizes the key role of diffusion phenomenon in the chloride ingress process in comparison with binding capacity and porosity of the concrete, as experimentally found by Pradelle et al. [70]. Our results were affected by bias calculations particularly for estimated Sobol indices close to 0 .

A comparison can be established with deterministic [13] and probabilistic SA [71] performed on diffusion-based corrosion initiation model by considering four governing parameters: apparent chloride diffusion, position of steel rebar, surface chloride concentration, critical chloride concentration. An approximation of the considered model was developed by applying analytical differential techniques based on a Taylor series that do not take into account pdf of parameters [13]. Their results are in agreement with ours, on

Table 2

Sensitivity analysis (Sobol and Morris indices) for input parameters involved in chloride transport model. Mean value of Sobol first order indices are highlighted in grey.

\begin{tabular}{|c|c|c|c|c|c|c|c|c|c|}
\hline \multirow[b]{3}{*}{ Parameters } & \multicolumn{6}{|c|}{ Sobol indices } & & \multicolumn{2}{|c|}{ Morris indices } \\
\hline & \multicolumn{6}{|c|}{ Indices of first order $S_{i}(\%)$ and second order $S_{i j}(\%)$} & \multirow{2}{*}{$\begin{array}{l}\text { Total order indices } \\
S T_{i}(\%)\end{array}$} & \multirow{2}{*}{$\begin{array}{l}\text { Algebraic } \\
\text { sign of } \mu\end{array}$} & \multirow{2}{*}{$\begin{array}{l}\text { Ratio } \\
\sigma_{i} / \mu_{i}^{*}\end{array}$} \\
\hline & $D_{e}$ & $C_{s}$ & $x_{\text {rebar }}$ & $\alpha$ & $C_{\text {crit }}$ & $p$ & & & \\
\hline$D_{e}$ & 29.90 & 15.38 & 11.63 & -0.18 & -0.39 & -0.61 & 70.45 & - & 2.02 \\
\hline$C_{s}$ & & 13.03 & 1.12 & 1.78 & 2.42 & -0.35 & 50.17 & - & 1.83 \\
\hline$x_{\text {rebar }}$ & & & 7.88 & -0.29 & -0.40 & -0.38 & 28.58 & + & 1.86 \\
\hline$\alpha$ & & & & 0.59 & -0.44 & -0.41 & 4.13 & + & 1.89 \\
\hline$C_{\text {crit }}$ & & & & & 0.50 & -0.36 & 21.41 & + & 2.66 \\
\hline$p$ & & & & & & 0.06 & 0.29 & - & 2.29 \\
\hline
\end{tabular}


the positive trend of $t_{c r i t}$ to $x_{\text {rebar }}$ and $C_{c r i t}$ and the negative trend of $t_{c r i t}$ to $C_{s}$ and $D_{e}$. However, the individual effects of parameters differ between the two studies. Different types of steels (conventional black carbon steel and corrosion resistant steels) and exposure conditions (light, moderate, high and severe) were considered in Ref. [13] such that the ranking of parameter importance varied between these different cases. In one case, the time $t_{c r i t}$ with regards to chloride diffusion was most sensitive to variations of $x_{\text {rebar }}$ then $D_{e}$; in other case, it was most sensitive to $C_{s}$ and $C_{\text {crit }}$. Importance factors of governing parameters were calculated in Ref. [71] by using their statistical distributions assumed to be lognormal. These factors were both influenced by time within the design life of the structure and coefficient of variation of parameters. According to these importance factors, the input parameter $x_{\text {rebar }}$ was ranked first, followed by $D_{e}, C_{s}$ and $C_{\text {crit }}$.

These results of SA cannot be entirely compared with ours since SA results must be interpreted with regards to variation range and statistical distributions considered for input parameters as well as the type of concrete structures studied. Furthermore, one of the main advantages of the approach applied in this work is the quantification of the influence of interaction between input parameters, which was not done by previous studies.

\subsubsection{Influences of interactions}

Possible interactions between parameters were indicated by the value of the ratio $\sigma_{\mathrm{i}} / \mu_{\mathrm{i}}^{*}$ superior to 0.5 for all parameters in Table 2 . Interactions detected by the Morris method are confirmed by the Sobol method. Results show that the influence of interactions between input parameters are the most important for $D_{e}$ and $C_{s}$ (via the calculations of the difference $S T_{i}-S_{i} \geq 30 \%$ ) and non-negligible for $x_{\text {rebar }}\left(S T_{i}-S_{i} \geq 20 \%\right)$. The strongest interactions is observed between $D_{e}$ and $C_{s}$ with $S_{i j}=15.38 \%$ then $D_{e}$ and $x_{\text {rebar }}$ with $S_{i j}=$ $11.63 \%$. Furthermore, significant interactions of third order (between three parameters) were also revealed by calculating $S T_{i}-S_{i}-$ $\sum_{j} S_{i j}$ shown to be superior to $10 \%$ for $D_{e}, C_{s}$ and $C_{c r i t}$.

Various bivariate graphs are shown below to highlight interactions. For sake of visibility, 1000 points were plotted in Figs. 2 and 3 , over the 5000 sample size used for simulation. As the parameter $D_{e}$ is found the most influential with a strong interaction with other parameters (Table 2), we investigated bivariate plots of $D_{e}$ as function of parameters such as $C_{s}$ (Fig. 2), $C_{c r i t}$ and $\alpha$ (Fig. 3). For instance, the time $t_{\text {crit }}$ (described by the size of points in Fig. 2) varies simultaneously according to $D_{e}$ and $C_{s}$, which emphasizes the strong interaction between these two parameters. In addition, around the mean value of $C_{s}=9.75 \mathrm{~kg} \cdot \mathrm{m}^{-3}, D_{e}$ ranges from $0.3 \times 10^{-12}$ to $5 \times$

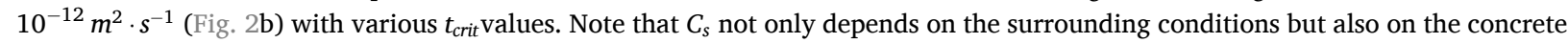
mix design reflected by the chloride binding capacity of the binder which in turn depends on its free chloride content and so on its diffusivity. This dependency between both parameters $D_{e}$ and $C_{s}$ was experimentally found by Othmen et al. [30] for total chloride profiles determined from 30 cores extracted at the same level above the sea from a reinforced concrete beam exposed to Atlantic ocean. The authors observed an increase of about 10 times in the diffusion coefficient for the mean chloride surface concentration (compared with about 16 times in Fig. 2b).

No interaction between $D_{e}$ and $C_{s}$, as well as between $D_{e}$ and $\alpha$ (Table 2) are found, and the time $t_{\text {crit }}$ only varies according to $D_{e}$ as shown in Fig. 3a and b. This trend is in accordance with the results obtained by Pradelle et al. on experimental data [70]. The weak effect of correlations between chloride diffusion coefficient, linear isotherm, and critical chloride content was globally highlighted through a sensitivity analysis: introducing correlations in the study will not modify the relative influence of each input data.

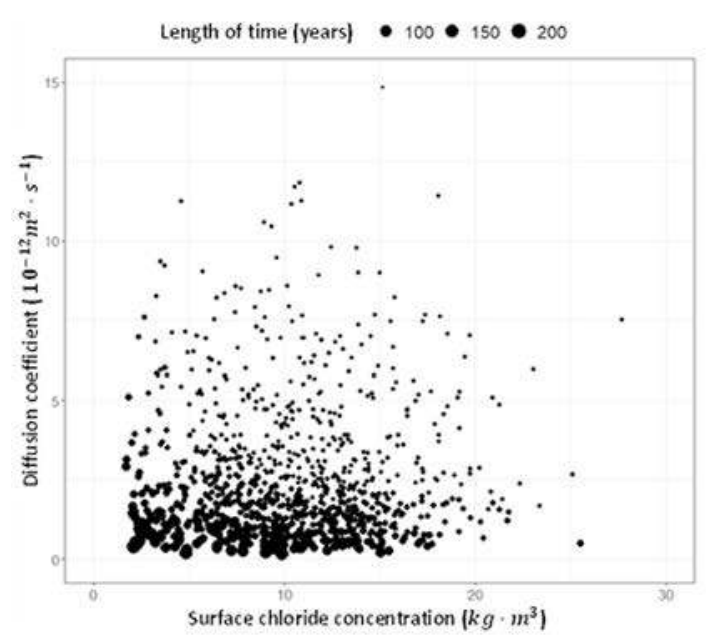

(a)

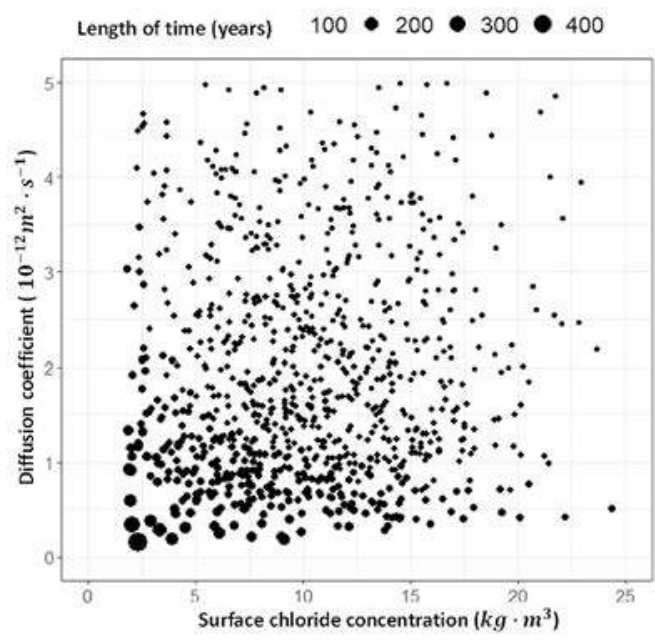

(b)

Fig. 2. Bivariate plot of (a) effective diffusion coefficient $D_{e}\left(10^{-12} \mathrm{~m}^{2} \cdot \mathrm{s}^{-1}\right)$ as function of surface chloride concentration $C_{s}\left(\mathrm{~kg} \cdot \mathrm{m}^{-3}\right)$ over 1000 simulated points, with (b) a zoom on interval ranges $D_{e}$ in $[0 ; 5]\left(10^{-12} \mathrm{~m}^{2} \cdot \mathrm{s}^{-1}\right)$ and $C_{s}$ in $[0 ; 25]\left(\mathrm{kg} \cdot \mathrm{m}^{-3}\right)$ over 883 simulated points. 


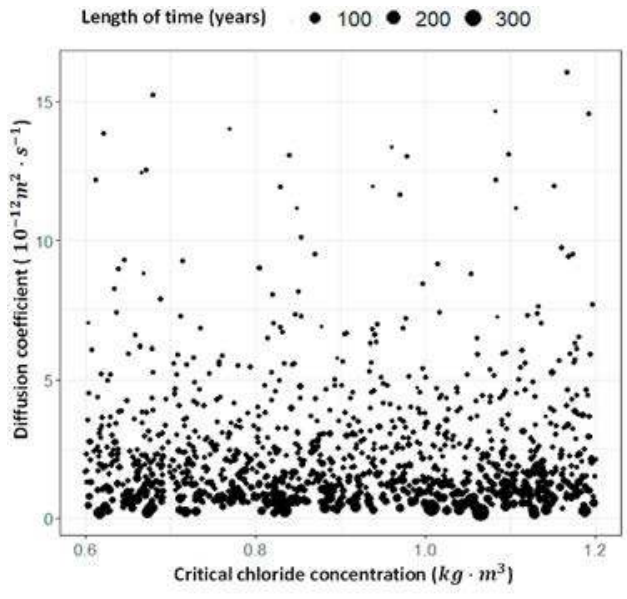

(a)

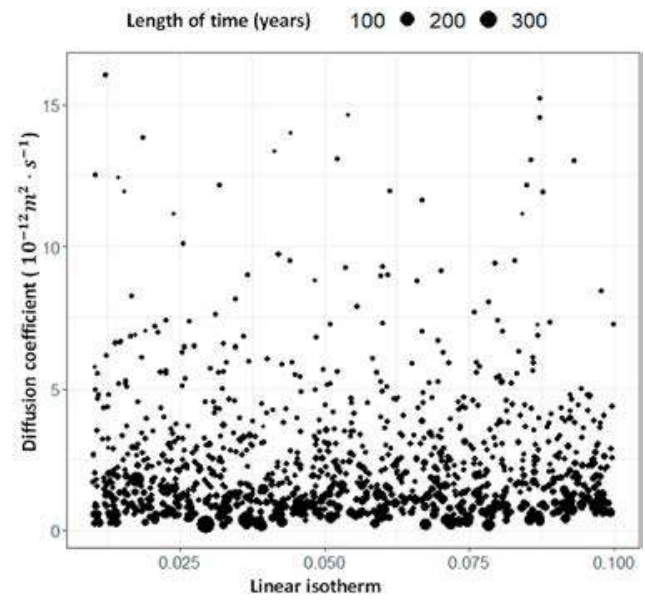

(b)

Fig. 3. Bivariate plot of effective diffusion coefficient $\left(10^{-12} \mathrm{~m}^{2} \cdot \mathrm{s}^{-1}\right)$ as function of (a) critical chloride content $\left(\mathrm{kg} \cdot \mathrm{m}^{-3}\right)$ and (b) linear isotherm over 1000 simulated points.

\subsection{Conditional probabilities of critical length of time given effective diffusion coefficient and surface chloride concentration}

For the influence of $D_{e}$ and $C_{s}$ pointed out from the analysis presented so far, the probabilistic analysis of $t_{c r i t}$ regarding cooccurrence of $D_{e}$ and $C_{s}$ was investigated by applying equation (8) given by

$$
\operatorname{Prob}\left(t_{c r i t} \geq t \mid D_{e} \geq \mathrm{d}, C_{s} \geq \mathrm{c}\right)=\frac{\operatorname{Prob}\left(t_{c r i t} \geq t, D_{e} \geq \mathrm{d}, C_{s} \geq \mathrm{c}\right)}{\operatorname{Prob}\left(D_{e} \geq \mathrm{c}, C_{s} \geq \mathrm{c}\right)},
$$

where $\mathrm{d}$ and $\mathrm{c}$ are predefined values of $D_{e}$ and $C_{s}$, respectively. According to the methodology described in Sub-section 2.2.2, conditional probabilities of $t_{\text {crit }}$ were calculated given the co-occurrence of both $D_{e}$ and $C_{s}$ by fixing other parameters $x_{\text {rebar }}, C_{\text {crit }}, p$ and $\alpha$ at their mean value. For instance, considering the case in which one hundred $C_{s}$ values (generated according to their pdf) were associated with one minimum $D_{e}=0.1 \times 10^{-12} \mathrm{~m}^{2} \cdot \mathrm{s}^{-1}$, the probability of $t_{\text {crit }}$ exceeding 15 years given $D_{e}$ and each $C_{s}$ was about $19-41 \%$ (Fig. 4a, c and Table 3). That probability logically decreased when $C_{s}$ increased. Likewise, considering one hundred $D_{e}$ values (generated according to their pdf) associated with the mean value of $C_{s}=9.75 \mathrm{~kg} \cdot \mathrm{m}^{-3}$, the probability of $t_{\text {crit }}$ exceeding 15 years was lied between 0 and $38 \%$ (Fig. $4 \mathrm{~b}, \mathrm{~d}$ and Table 3). Therefore, to achieve an extension of $t_{\text {crit }}$ exceeding 15 years was more reliable with $D_{e}=0.1 \times$ $10^{-12} \mathrm{~m}^{2} \cdot \mathrm{s}^{-1}$ and various $C_{s}$ than with $C_{s}=9.75 \mathrm{~kg} \cdot \mathrm{m}^{-3}$ and various $D_{e}$. In addition, the conditional probabilities of $t_{\text {crit }} \mathrm{decreased}$ more quickly as $D_{e}$ increased than as $C_{s}$ increased. Thus, the extension of $t_{\text {crit }}$ was combined with a decreasing probability of its occurrence.

\subsection{Comparisons of simulated scenarios}

To deeply investigate the time $t_{\text {crit }}$ as function of $D_{e}$ from conditional probabilities calculated so far, three scenarios were simulated: a medium scenario in which $D_{e}$ was set to its mean value, and a favorable and unfavorable scenarios in which $D_{e}$ was set to its minimum and maximum values, respectively. In the three scenarios, technological parameters $x_{r e b a r}, p$ and $\alpha$ were set at their mean value and surrounding parameters $C_{s}$ and $C_{\text {crit }}$ according to their pdf. Probability distributions of simulated critical length of time to corrosion initiation values were plotted in Fig. 5 and Table 4 presents mean and standard deviation of the simulated values of $t_{\text {crit }}$ for the three scenarios. The comparison between favorable and medium scenarios indicates that the corrosion initiation time can be extended by 12 through a decrease of $D_{e}$. However, this improvement is coupled with a large increase of the uncertainty affecting the reliability of the service life extension of $t_{c r i t}$ in the favorable scenario. That is consistent with conditional probabilities of $t_{c r i t}$ previously showed in Fig. 4. Conversely, the comparison between unfavorable and medium scenarios indicates that initiation corrosion time is 3 times reduced by an increase of $D_{e}$ coupled with a reduction of the uncertainty. This means a better reliability of the average length of time to corrosion initiation in the unfavorable scenario. In addition, a scenario corresponding to an average value of $t_{\text {crit }}$ close to 100 years (95 years in mean with a standard deviation of 60.6 years) associated with a $D_{e}=0.25 \times 10^{-12} \mathrm{~m}^{2} \cdot \mathrm{s}^{-1}$ was presented as a reference for service life behavior of structures for sector's actor.

\section{Concluding remarks}

This study has pointed out the influential role of both parameters related to material properties and surrounding conditions, and their interactions, on the reliability of results from a chloride transport model. Sensitivity Analysis (SA) and conditional probabilities of 


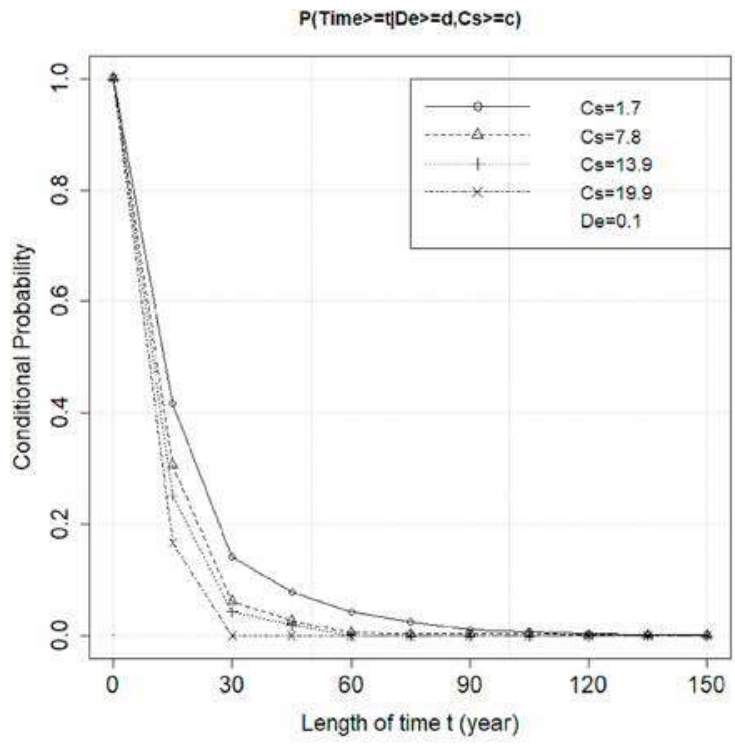

(a)

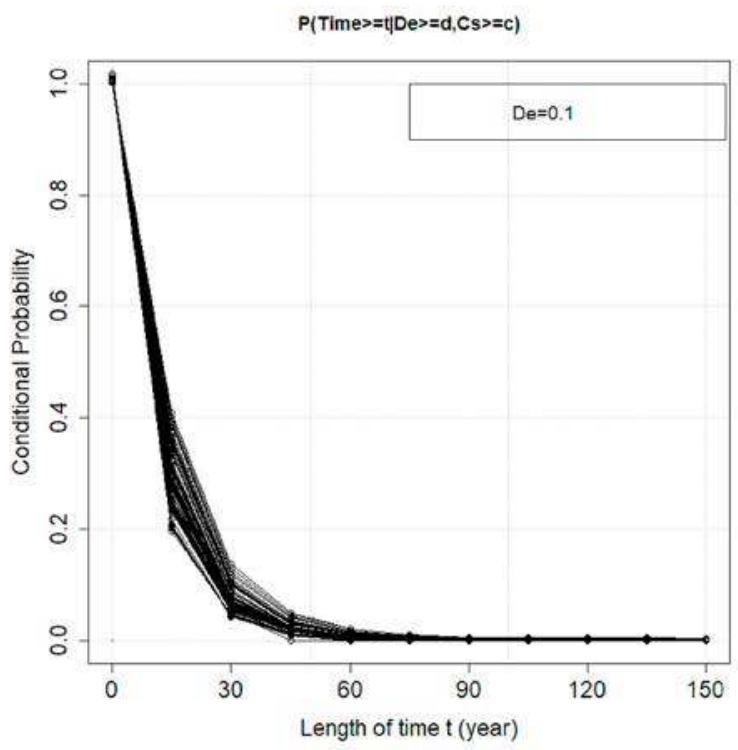

(c)

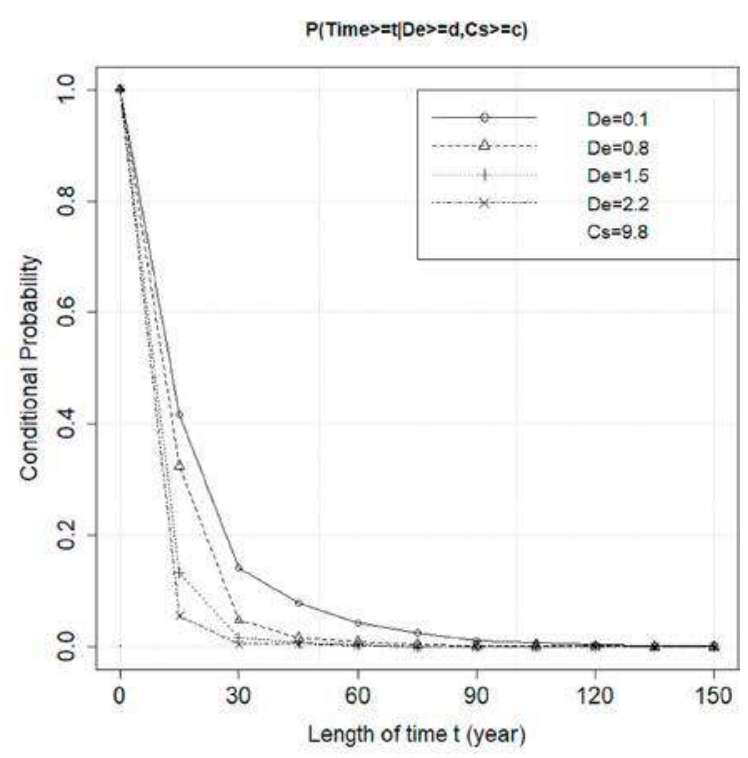

(b)

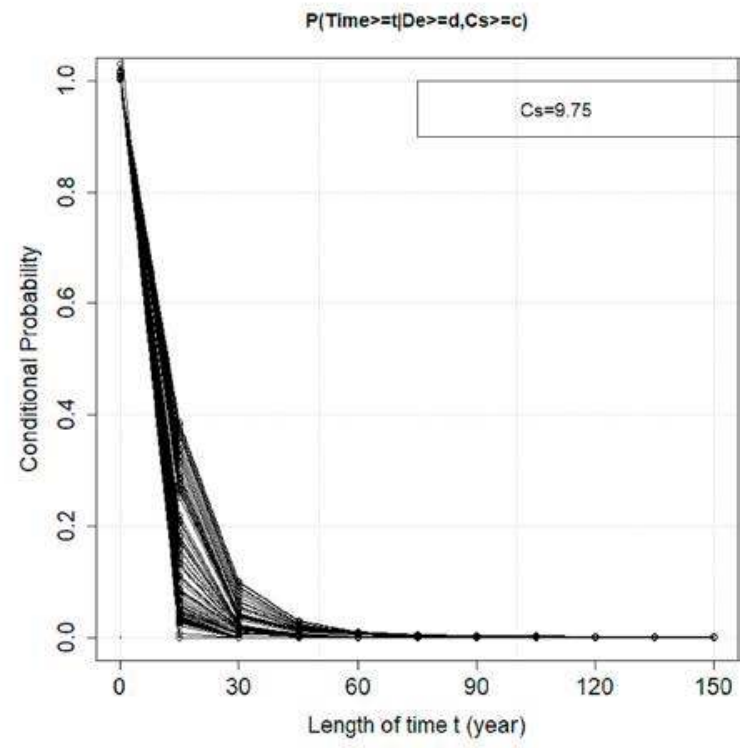

(d)

Fig. 4. Conditional probabilities of the critical length of time $t$ (year) to corrosion initiation given the co-occurrence of effective diffusion coefficient $\mathrm{D}_{\mathrm{e}}=0.1 \times 10^{-12} \mathrm{~m}^{2} \cdot \mathrm{s}^{-1}$ and (a) four and (c) one hundred values of surface chloride concentration $\left(\mathrm{C}_{\mathrm{s}}, \mathrm{kg} \cdot \mathrm{m}^{-3}\right)$, and given the co-occurrence of $\mathrm{C}_{\mathrm{s}}$ $=9.75 \mathrm{~kg} \cdot \mathrm{m}^{-3}$ and (b) four and (d) one hundred values of $\mathrm{D}_{\mathrm{e}}$.

length of time to corrosion initiation have been used in a probabilistic approach to investigate the corrosion risk due to chloride ingress into RC structures.

The SA has shown that the key input data for predicting time to corrosion initiation are clearly the chloride diffusion coefficient, the surface chloride concentration and the position of steel rebar. The only controllable parameters are the effective diffusion coefficient and concrete cover thickness. It is crucial to define with enough accuracy the mean value and the coefficient of variation of the pdf of these input data. The diffusion chloride coefficient is particularly an action lever that enables relevant stakeholders to influence the service life behavior of structures, in comparison to binding capacity and porosity of the concrete. That finally allows limiting expensive and time consuming experimental tests, necessary to determine input data to predict service life of reinforced concrete structures in marine environment. In a further work, it would be interesting to take into account the time dependency of the diffusion 
Table 3

Variation ranges of conditional probability of the critical length of time $t_{\text {crit }}$ to corrosion initiation given (a) the co-occurrence of effective diffusion coefficient $\mathrm{D}_{\mathrm{e}}=0.1 \times 10^{-12} \mathrm{~m}^{2} \cdot \mathrm{s}^{-1}$ and one hundred values of surface chloride concentration $\mathrm{C}_{\mathrm{s}}\left(\mathrm{kg} \cdot \mathrm{m}^{-3}\right)$, and (b) the cooccurrence of $\mathrm{C}_{\mathrm{s}}=9.75 \mathrm{~kg} \cdot \mathrm{m}^{-3}$ and one hundred values of $\mathrm{D}_{\mathrm{e}}$.

\begin{tabular}{lll}
\hline Length of time $t$ (year) & Variation range of conditional probability of $t_{\text {crit }}(\%)$ & \\
\cline { 2 - 3 } & (a):Prob $\left(t_{\text {crit }} \geq t \mid D_{e} \geq 0.1, C_{s} \geq c\right)$ & (b):Prob $\left(t_{\text {crit }} \geq t \mid D_{e} \geq d, C_{s} \geq 9.75\right)$ \\
\hline 10 & {$[20 ; 65]$} & {$[0 ; 64]$} \\
15 & {$[19 ; 41]$} & {$[0 ; 38]$} \\
30 & {$[4 ; 14]$} & {$[0 ; 10]$} \\
38 & {$[0 ; 10]$} & {$[0 ; 8]$} \\
45 & {$[0 ; 5]$} & {$[0 ; 3]$} \\
60 & {$[0 ; 2]$} & {$[0,1]$} \\
\hline
\end{tabular}

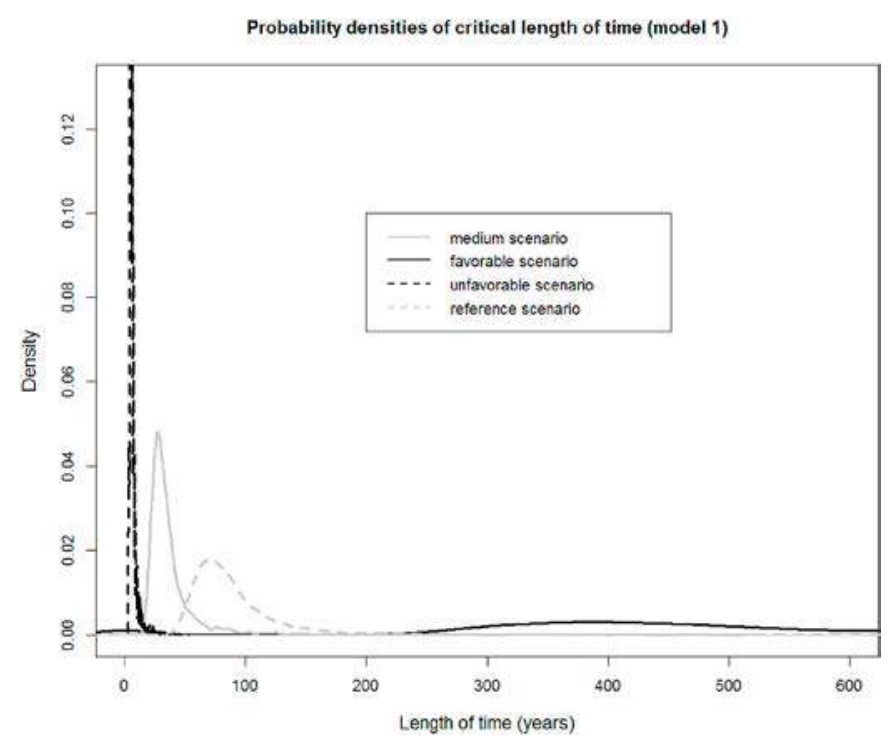

Fig. 5. Pdf of the critical length of time (year) to corrosion initiation for 4 scenarios: unfavorable (with $D_{e}=6.0 \times 10^{-12} \mathrm{~m}^{2} \cdot \mathrm{s}^{-1}$ ), medium (with $D_{e}$ $=0.66 \times 10^{-12} \mathrm{~m}^{2} \cdot \mathrm{s}^{-1}$ ), reference (with $D_{e}=0.25 \times 10^{-12} \mathrm{~m}^{2} \cdot \mathrm{s}^{-1}$ ) and favorable (with $D_{e}=0.1 \times 10^{-12} \mathrm{~m}^{2} \cdot \mathrm{s}^{-1}$ ).

Table 4

Descriptive statistics (mean and standard deviation) of pdf of the critical length of time to corrosion initiation for various scenarios.

\begin{tabular}{llll}
\hline Scenarios with $x_{\text {rebar }}=50 \mathrm{~mm}$ and $p=12.85 \%$ & $D_{e}\left(10^{-12} \mathrm{~m}^{2} \cdot \mathrm{s}^{-1}\right)$ & Mean (years) & Standard deviation (years) \\
\hline Unfavorable: maximum $D_{e}$ & 6 & 10 & 6.1 \\
Medium: mean $D_{e}$ & 0.66 & 38 & 27.1 \\
Reference: $D_{e}$ calculated to obtain the critical length of time 100 years & 0.25 & 100 & 60.6 \\
Favorable: minimum $D_{e}$ & 0.1 & 439 & 184.8 \\
\hline
\end{tabular}

coefficient by introducing an ageing factor.

An emphasis is also shown on the effect of interaction between parameters $D_{e}$ and $C_{s}$. By acting on controllable parameter $D_{e}$ to extend the time at which chloride concentration achieve a critical level requires to consider possible variations in surrounding parameters, such as chloride concentration at the concrete surface. These interactions emphasize the fact that length of time to corrosion initiation results from complex phenomena concerning the sea water and concrete structures and not only from individual influence of transport and chemical properties of concrete materials. SA methods that focus on combined influences of groups (triplets, ...) of input parameters should be deeper investigated.

The results of conditional probabilities of length of time $t_{\text {crit }}$ to corrosion initiation in regard to the influence from the co-occurrence of $D_{e}$ and $C_{s}$ have contributed to the reliability assessment of extension of $t_{\text {crit }}$. The sector's actor faces the choice either of extending the service life of structures but with a lower reliability of $t_{\text {crit }}$ 's extension or of getting a lowest quality of service life but with a higher reliability of $t_{\text {crit's }}$ reduction. 


\section{Declaration of competing interest}

The authors declare that they have no known competing financial interests or personal relationships that could have appeared to influence the work reported in this paper.

\section{Acknowledgements}

The research and education chair of civil engineering and eco-construction is financed by the Trade and Industry Chamber of Nantes and Saint-Nazaire cities (France), the urban community of Saint-Nazaire (France), Charier Company (Montoir, France), Architectes Ingénieurs Associés (AIA) (Nantes, France), Vinci construction France, the French Federation of Buildings and the Regional Federation of Public Works. The authors wish to thank these partners for their patronage. We also thank the two anonymous referees for their careful review and helpful comments, which led to improve the article.

\section{Appendix A. Supplementary data}

Supplementary data to this article can be found online at https://doi.org/10.1016/j.marstruc.2020.102804.

\section{References}

[1] Baroghel-Bouny V. Concrete design for structures with predefined service life - durability control with respect to reinforcement corrosion and alkali-silica reaction state-of-the-art and guide for the implementation of a performance-type and predictive approach based upon durability indicators. English version of Documents Scientifiques et Techniques de l'AFGC (Civil Engineering French Association); 2004.

[2] Tuutti K. Corrosion of steel in concrete. Stockholm: Swedish Cement and Concrete Research Institute; 1982.

[3] BSEN1504-9. Products and systems for the protection and repair of concrete structures. Definitions, requirements, quality control and evaluation of conformity. General principles for use of products and systems. 2008.

[4] WBCSD IEA. Cement Technology Roadmap 2009: carbon emissions reductions up to 2050. World Business Council for Sustainable Development and International Energy Agency; 2009.

[5] Bastidas-Arteaga E, Chateauneuf A, Sánchez-Silva M, Bressolette P, Schoefs F. A comprehensive probabilistic model of chloride ingress in unsaturated concrete. Eng Struct 2011;33:720-30.

[6] Ryan PC, O'Connor AJ. Probabilistic analysis of the time to chloride induced corrosion for different Self-Compacting Concretes. Construct Build Mater 2013;47: $1106-16$.

[7] de Vera G, Antón C, López MP, Climent MA. Depassivation time estimation in reinforced concrete structures exposed to chloride ingress: a probabilistic approach. Cement Concr Compos 2017;79:21-33.

[8] Duprat F, Sellier A. Probabilistic approach to corrosion risk due to carbonation via an adaptive response surface method. Probabilist Eng Mech 2006;21:207-16.

[9] Van-Loc TA, Senga Kiesse T, Bonnet S, Ventura A. Application of sensitivity analysis in the life cycle design for the durability of reinforced concrete structures in the case of XC4 exposure class. Cement Concr Compos 2018;87:53-62.

[10] Tran T-B, Bastidas-Arteaga E, Schoefs F, Bonnet S. A Bayesian network framework for statistical characterisation of model parameters from accelerated tests: application to chloride ingress into concrete. Structure and Infrastructure Engineering 2018;14:580-93.

[11] Boddy A, Bentz E, Thomas MDA, Hooton RD. An overview and sensitivity study of a multimechanistic chloride transport model. Cement Concr Res 1999;29: $827-37$.

[12] Kirkpatrick TJ, Weyers RE, Anderson-Cook CM, Sprinkel MM. Probabilistic model for the chloride-induced corrosion service life of bridge decks. Cement Concr Compos 2002;32:1943-60.

[13] Zhang J, Lounis Z. Sensitivity analysis of simplified diffusion-based corrosion initiation model of concrete structures exposed to chlorides. Cement Concr Res 2006;36:1312-23.

[14] Prinja NK, Ogunbadejo A, Sadeghi J, Patelli E. Structural reliability of pre-stressed concrete containments. Nucl Eng Des 2017;323:235-44.

[15] Zhu F, Zhou Q, Wang F, Yang X. Spatial variability and sensitivity analysis on the compressive strength of hollow concrete block masonry wallettes. Construct Build Mater 2017;140:129-38.

[16] Morris D. Factorial sampling plans for preliminary computational experiments. Technometrics 1991;33:161-74.

[17] Sobol I. Global sensitivity indices for nonlinear mathematical models and their Monte Carlo estimates. Math Comput Simulat 2001;55:271-80.

[18] Samson E, Marchand J, Beaudoin JJ. Describing ion diffusion mechanisms in cement-based materials using the homogenization technique. Cement Concr Compos 1999;29:1341-5.

[19] M. Maage, S. Helland E. Poulson O. Vennesland. C. Jan Erik, Service life prediction of existing concrete structures exposed to marine environment, Materials Journal, 93.

[20] Audenaert K, Yuan Q, De Schutter G. On the time dependency of the chloride migration coefficient in concrete. Construct Build Mater 2010;24:396-402.

[21] Ababneh A, Benboudjema F, Xi Y. Chloride penetration in nonsaturated concrete. J Mater Civ Eng 2003;15:183-91.

[22] Anna RVS, Saetta V, Renato VV. Analysis of chloride diffusion into partially saturated concrete. Materials Journal $1993 ; 90$.

[23] Baroghel-Bouny V, Thiéry M, Wang X. Modelling of isothermal coupled moisture-ion transport in cementitious materials. Cement Concr Res 2011;41:828-41.

[24] Meijers SJH, Bijen JMJM, de Borst R, Fraaij ALA. Computational results of a model for chloride ingress in concrete including convection, drying-wetting cycles and carbonation. Mater Struct 2005;38:145-54.

[25] O'Neill Iqbal P, Ishida T. Modeling of chloride transport coupled with enhanced moisture conductivity in concrete exposed to marine environment. Cement Concr Res 2009;39:329-39.

[26] Samson E, Marchand J, Snyder KA, Beaudoin JJ. Modeling ion and fluid transport in unsaturated cement systems in isothermal conditions. Cement Concr Res 2005;35:141-53.

[27] Sleiman H, Amiri O, Ait-Mokhtar A, Loche J-M. Chloride transport through unsaturated concrete: chloride profile simulations and experimental validation. Mag Concr Res 2012;64:351-9.

[28] EN206-1, EN 206-1 Concrete - Part 1: specification, performances, production and conformity. 2004.

[29] EN1992-1-1, EN 1992-1-1 Eurocode 2 - design of Concrete structure - Part 1.1 General rules and rules for buildings. 2005.

[30] Othmen I, Bonnet S, Schoefs F. Statistical investigation of different analysis methods for chloride profiles within a real structure in a marine environment. Ocean Eng 2018;157:96-107.

[31] Chalee W, Jaturapitakkul C, Chindaprasirt P. Predicting the chloride penetration of fly ash concrete in seawater. Mar Struct 2009;22:341-53. 
[32] Tadayon MH, Shekarchi M, Tadayon M. Long-term field study of chloride ingress in concretes containing pozzolans exposed to severe marine tidal zone. Construct Build Mater 2016;123:611-6.

[33] Valipour M, Pargar F, Shekarchi M, Khani S, Moradian M. In situ study of chloride ingress in concretes containing natural zeolite, metakaolin and silica fume exposed to various exposure conditions in a harsh marine environment. Construct Build Mater 2013;46:63-70.

[34] Real S, Bogas JA. Chloride ingress into structural lightweight aggregate concrete in real marine environment. Mar Struct 2018;61:170-87.

[35] Amiri O, Aït-Mokhtar A, Seigneurin A. A complement to the discussion of Xu A., Chandra S., calculation of chloride diffusion coefficients in concrete from ionic migration measurements by Andrade C. Cement Concr Compos 1997;27:951-7.

[36] Crank. The mathematics of diffusion. second ed. ed. Oxford: Clarendon Press; 1975.

[37] Ben Fraj A, Bonnet S, Khelidj A. New approach for coupled chloride/moisture transport in non-saturated concrete with and without slag. Construct Build Mater $2012 ; 35: 761-71$.

[38] Martin-Pérez B, Zibara H, Hooton RD, Thomas MDA. A study of the effect of chloride binding on service life predictions. Cement Concr Compos 2000;30: $1215-23$.

[39] Code CFFM. Model code for service life design. Lausanne: Federation Internationale du Beton, fib, Bulletin; 2006.

[40] EuRam III EB. DuraCrete final technical report, probabilistic performance based durability design of concrete structures. 2000. Document BE95-1347.

[41] Tran VQ, Soive A, Bonnet S, Khelidj A. A numerical model including thermodynamic equilibrium, kinetic control and surface complexation in order to explain cation type effect on chloride binding capability of concrete. Construct Build Mater 2018;191:608-18.

[42] Li D, Li L-y, Wang X. Chloride diffusion model for concrete in marine environment with considering binding effect. Mar Struct 2019;66:44-51.

[43] Yuan Q, Shi C, De Schutter G, Audenaert K, Deng D. Chloride binding of cement-based materials subjected to external chloride environment - a review. Construct Build Mater 2009;23:1-13.

[44] Song Z, Jiang L, Zhang Z, Xiong C. Distance-associated chloride binding capacity of cement paste subjected to natural diffusion. Construct Build Mater 2016;112: 925-32.

[45] Mohammed TU, Hamada H. Relationship between free chloride and total chloride contents in concrete. Cement Concr Compos 2003;33:1487-90.

[46] Cheewaket T, Jaturapitakkul C, Chalee W. Long term performance of chloride binding capacity in fly ash concrete in a marine environment. Construct Build Mater 2010;24:1352-7.

[47] Otieno MB, Beushausen HD, Alexander MG. Modelling corrosion propagation in reinforced concrete structures - a critical review. Cement Concr Compos 2011; $33: 240-5$.

[48] Andrade C, Castellote M. Analysis of total chloride content in concrete. Mater Struct 2002;35:583-5.

[49] Alonso C, Andrade C, Castellote M, Castro P. Chloride threshold values to depassivate reinforcing bars embedded in a standardized OPC mortar. Cement Concr Res 2000;30:1047-55.

[50] Gouda VK. Corrosion and corrosion inhibition of reinforcing steel: I. Immersed in Alkaline solutions. Br Corrosion J 1970;5:198-203.

[51] Angst U, Elsener B, Larsen CK, Vennesland Ø. Critical chloride content in reinforced concrete — a review. Cement Concr Res 2009;39:1122-38.

[52] G.P. Radhakrishna, T. David, Surface condition effects on critical chloride threshold of steel reinforcement, Materials Journal, 102.

[53] Ghods P, Isgor OB, Brown JR, Bensebaa F, Kingston D. XPS depth profiling study on the passive oxide film of carbon steel in saturated calcium hydroxide solution and the effect of chloride on the film properties. Appl Surf Sci 2011;257:4669-77.

[54] Ann KY, Song H-W. Chloride threshold level for corrosion of steel in concrete. Corrosion Sci 2007;49:4113-33.

[55] Glass GK, Buenfeld NR. The presentation of the chloride threshold level for corrosion of steel in concrete. Corrosion Sci 1997;39:1001-13.

[56] Andrianandraina A Ventura, Kiessé T Senga, Cazacliu B, Idir R, van der Werf HMG. Sensitivity analysis of environmental process modeling in a life cycle context: a case study of hemp crop production. J Ind Ecol 2015;19:978-93.

[57] Ventura A, Idir R, Marceau S, van Schoors L, Andrianandraina, van der Werf HMG. How to use LCA to assess materials as eco-design parameters in construction projects. In: International symposium on life cycle assessment and construction. France: Civil engineering and buildings; 2012. A. Ventura and C. de la RocheNantes.

[58] Castellote M, Andrade C, Alonso C. Measurement of the steady and non-steady state chloride diffusion coefficients in a migration test by means of monitoring the conductivity in the anolyte chamber, Comparison with natural diffusion tests. Cement Concr Compos 2001;31:1411-20.

[59] Djerbi A, Bonnet S, Khelidj A, Baroghel-bouny V. Influence of traversing crack on chloride diffusion into concrete. Cement Concr Res 2008;38:877-83.

[60] Djerbi Tegguer A, Bonnet S, Khelidj A, Baroghel-Bouny V. Effect of uniaxial compressive loading on gas permeability and chloride diffusion coefficient of concrete and their relationship. Cement Concr Res 2013;52:131-9.

[61] Jang SY, Kim BS, Oh BH. Effect of crack width on chloride diffusion coefficients of concrete by steady-state migration tests. Cement Concr Res 2011;41:9-19.

[62] Rozière E, Loukili A, Cussigh F. A performance based approach for durability of concrete exposed to carbonation. Construct Build Mater 2009;23:190-9.

[63] Tang L, Nilsson L-O. Chloride binding capacity and binding isotherms of OPC pastes and mortars. Cement Concr Compos 1993;23:247-53.

[64] Tang L, Sørensen HE. Precision of the Nordic test methods for measuring the chloride diffusion/migration coefficients of concrete. Mater Struct 2001;34:479-85.

[65] Tong L, Gjørv OE. Chloride diffusivity based on migration testing. Cement Concr Compos 2001;31:973-82.

[66] Truc O, Ollivier JP, Carcassès M. A new way for determining the chloride diffusion coefficient in concrete from steady state migration test. Cement Concr Compos 2000;30:217-26.

[67] Yang CC. A comparison of transport properties for concrete using the ponding test and the accelerated chloride migration test. Mater Struct 2005;38:313-20.

[68] Duprat F. Reliability of RC beams under chloride-ingress. Construct Build Mater 2007;21:1605-16.

[69] Bastidas-Arteaga E, Chateauneuf A, Sánchez-Silva M, Bressolette P, Schoefs F. Influence of weather and global warming in chloride ingress into concrete: a stochastic approach. Struct Saf 2010;32:238-49.

[70] Pradelle S, Thiéry M, Baroghel-Bouny V. Sensitivity analysis of chloride ingress models: case of concretes immersed in seawater. Construct Build Mater 2017; 136:44-56.

[71] Saassouh B, Lounis Z. Probabilistic modeling of chloride-induced corrosion in concrete structures using first- and second-order reliability methods. Cement Concr Compos 2012;34:1082-93. 